\title{
THE PRINCIPAL SEMI-ALGEBRA IN A BANACH ALGEBRA $\left({ }^{1}\right)$
}

\author{
BY \\ EDWARD J. BARBEAU \\ INTRODUCTION
}

0.1. Orientation. A subset of a real linear associative algebra is called a semi-algebra iff it is a wedge $[8$, p. 20] closed under multiplication. The theory of such a structure has been developed principally by F. F. Bonsall, who originally considered semi-algebras of non-negative-valued functions, but later extended his research to include locally compact and convolution semi-algebras [1], [2], [3]. Independently, S. Bourne [6] defined semi-algebras and proved a representation theorem for a special class of them.

The present paper carries the study in a new direction. The question of determining maximal closed subalgebras of certain Banach algebras has received a great deal of attention (see, for example, [10]), but the corresponding problem for semi-algebras seems not to have been considered at all. That some interesting results might be obtained was suggested by the observation that, with real Euclidean 3-space considered naturally as a Banach algebra (operations defined pointwise), the solid $\left\{(x, y, z): x, y, z \geqq 0, z \leqq x^{1 / 2} y^{1 / 2}\right\}$ is a maximal closed subsemi-algebra of the positive octant. I am grateful to Professor L. Nachbin, who, by conjecturing a possible generalization for continuous real-valued functions, led me into a deeper consideration of the problem, and to Professor F. F. Bonsall for his encouragement and supervision.

The setting for Chapter 1 is a real Banach algebra with multiplicative identity 1 . The role of the positive octant in the above example is played by the principal semi-algebra, defined to be the least closed semi-algebra whose interior contains 1 . A representation of this semi-algebra is given, along with some results and examples concerning its maximal closed subsemi-algebras. Chapter 2 deals with the particular Banach algebra of all continuous real-valued functions defined on a compact Hausdorff space, whose principal semi-algebra is the set of all its non-negative-valued functions. In this case, not only can all maximal

Received by the editors November 27, 1964.

(1) The results in this paper are contained in the author's doctoral thesis, prepared while he was a Commonwealth Scholar at the University of Newcastle-upon-Tyne in the United Kingdom. 
closed subsemi-algebras of the principal semi-algebra be found, but a simple characterization of all their intersections can be given.

The paper which is probably most closely related to the results of Chapter 2 is one by W. B. Jurkat and G. G. Lorentz [11], which gives necessary and sufficient conditions involving simple geometric means that a pair of functions generate the uniformly closed semi-algebra of non-negative continuous functions on a compact Hausdorff space. It seems that it may be possible to obtain this result as part of the present theory. I note also that geometric means of functions have arisen in work by D. G. Bourgin [5] and J. F. C. Kingman [13].

The remainder of the introduction contains a list of results for later reference.

0.2. The geometric mean of a non-negative continuous function. Here, and throughout the paper, $E$ denotes a compact Hausdorff space, $C(E)$ the Banach algebra (with algebraic operations defined pointwise and the uniform norm) of all continuous real-valued functions defined on $E, C^{+}(E)$ the set of all nonnegative functions in $C(E)$ and $C^{\#}(E)$ the set of all those functions in $C(E)$ which are everywhere strictly positive. Consider $C(E)$ as being ordered with positive cone $C^{+}(E)$. See N. Bourbaki [4] for background in integration theory.

Let $\mu$ be a Radon probability measure on $E$ (i.e. a positive measure of total mass 1). The geometric mean with weight $\mu$, denoted by $G M_{\mu}$, is defined on $C^{+}(E)$ by

$$
G M_{\mu} f= \begin{cases}\exp \int \log f d \mu & \text { if } \log f \text { is } \mu \text {-integrable } \\ 0 & \text { if } \log f \text { is not } \mu \text {-integrable. }\end{cases}
$$

(Note that $\log f$ may be an extended real-valued function.) For $f \in C^{\#}(E), \log f$ is $\mu$-integrable and the first alternative of the definition is valid. Observe that, if the support of $\mu$ is a finite subset of $E$, then $G M_{\mu} f$ is expressible as a finite product.

$G M_{\mu}$ is a non-negative monotone function on $C^{+}(E)$ with the following properties (where $f, g \in C^{+}(E), \lambda \geqq 0$ ):

(a) $G M_{\mu} \lambda=\lambda$;

(b) $\lim _{\lambda \rightarrow 0^{+}} G M_{\mu}(f+\lambda g)=G M_{\mu} f$;

(c) $0 \leqq G M_{\mu} f \leqq \mu(f) \leqq\|f\|$;

(d) $G M_{\mu} f g=\left(G M_{\mu} f\right)\left(G M_{\mu} g\right) ; G M_{\mu} \lambda f=\lambda G M_{\mu} f ; G M_{\mu}(f+g) \geqq G M_{\mu} f+G M_{\mu} g$; $G M_{\mu} f^{\lambda}=\left(G M_{\mu} f\right)^{\lambda}$.

The proofs are straightforward. For (b), because of the monotonicity of $G M_{\mu}$, it suffices to consider the integrals of the functions in the decreasing sequence $\left\{\log \left(f+n^{-1} g\right)\right\}$ with pointwise limit $\log f$. If the sequence contains any non$\mu$-integrable member, or if the integrals are not bounded below, then $\log f$ is not $\mu$-integrable. Otherwise, an application of the Beppo Levi Theorem [4, pp. 138, 149] yields the result. Since the second inequality of (c) and the superadditivity property in (d) are not quite obvious, a proof following that in $[9$, p. 138] is out- 
lined. When $\mu(f)>0$ and $\log f$ is $\mu$-integrable, it follows from the positivity of $\mu$ and the inequality $\log \beta \leqq \beta-1$ for positive real $\beta$, that

$$
\begin{aligned}
\int \log f d \mu & =\int \log (f / \mu(f)) d \mu+\log \int f d \mu \\
& \leqq \int[(f / \mu(f))-1] d \mu+\log \int f d \mu=\log \int f d \mu
\end{aligned}
$$

For the remaining cases, note that, for positive $\lambda, G M_{\mu}(f+\lambda) \leqq \mu(f+\lambda)$ and use (b) and the continuity of $\mu$. To show the superadditivity of $G M_{\mu}$, observe that when $f+g \in C^{*}(E)$,

$$
G M_{\mu}(f /(f+g))+G M_{\mu}(g /(f+g)) \leqq \mu(f /(f+g))+\mu(g /(f+g))=1 ;
$$

when $f+g$ has a zero, approximate to $f$ by $f+\lambda$.

Finally, when $\mu_{1}$ and $\mu_{2}$ are probability measures on $E$ and $\mu=\alpha \mu_{1}+(1-\alpha) \mu_{2}$ for $0<\alpha<1$, then, for each $f \in C^{+}(E)$,

$$
G M_{\mu} f=\left(G M_{\mu_{1}} f\right)^{\alpha} \cdot\left(G M_{\mu_{2}} f\right)^{1-\alpha} \text {. }
$$

0.3. The theorem of Choquet and Deny. A subset $P$ of $C(E)$ is an upper semilattice iff, whenever $f, g \in P$, it is true that $f \cup g \in P$, where

$$
(f \cup g)(\xi) \equiv \max \{f(\xi), g(\xi)\} \quad(\xi \in E) .
$$

Let $\mu$ be a positive Radon measure on $E$ and $\xi$ be a point of $E$. Then the sets

$$
\begin{aligned}
U_{\mu, \xi} & \equiv\{f: f \in C(E), \mu(f) \geqq f(\xi)\}, \\
U_{\mu} & \equiv\{f: f \in C(E), \mu(f) \geqq 0\}
\end{aligned}
$$

are both uniformly closed wedges which are upper semi-lattices. The following theorem states that every upper semi-lattice uniformly closed wedge is the intersection of wedges of the types $U_{\mu, \xi}$ and $U_{\mu}$. Let $\delta_{\xi}$ denote the measure with all of its unit mass concentrated at the point $\xi$, and $W^{\prime}$ denote the dual wedge of all continuous real linear functionals taking non-negative values on the wedge $W$.

THEOREM 0.1 (CHOQUeT-DENY). Let $W$ be a uniformly closed wedge contained in $C(E)$ which is an upper semi-lattice. Suppose that $\mathscr{U}_{1}$ is the family of all pairs $(\mu, \xi)$ with $\xi \in E$ and $\mu$ a positive Radon measure such that $\mu(\{\xi\})=0$ and $\mu-\delta_{\xi} \in W^{\prime}$; suppose that $\mathscr{U}_{2}$ is the family of all positive Radon measures $\mu$ such that $\mu \in W^{\prime}$. Then:

$$
W=\left(\bigcap_{(\mu, \xi) \in \mathscr{U}_{1}} U_{\mu, \xi}\right) \cap\left(\bigcap_{\mu \in \mathscr{U}_{2}} U_{\mu}\right) .
$$

For the proof, see [7]. The theorem is valid even if $\mathscr{U}_{1}$ is void and $\mathscr{U}_{2}$ consists of the zero measure alone. (The convention that a void intersection is the whole of the space is adopted.) 


\section{CHAPTER 1}

1.1. The principal semi-algebra. Throughout this chapter, $B$ is a real Banach algebra with multiplicative identity 1 . Denote by $G$ the set of regular elements of $B$. The space $\Phi_{B}^{c}$ of nontrivial complex homomorphisms on $B$ is compact in the Gelfand topology; the family $\Phi_{B}$ of all nontrivial real homomorphisms is a closed subset of $\Phi_{B}^{c}$. A nonvoid subset $A$ of $B$ is a semi-algebra iff

$$
x, y \in A, \lambda \geqq 0 \Rightarrow x+y, x y, \lambda x \in A .
$$

Denote by $B^{*}$ the dual space consisting of all continuous real linear functionals on $B$, by $A^{\prime}$ the subset of those functionals in $B^{*}$ taking non-negative values on $A$. Let $\boldsymbol{R}$ be the set of reals, $\boldsymbol{R}^{+}$the set of non-negative reals. The proof of the following theorem is similar to the proof of a theorem given by Kadison in $[12$, p. 8$]$.

THEOREM 1.1. Let $A$ be any closed subsemi-algebra of $B$ whose interior contains 1. Then $A=\bigcap_{\phi \in \Psi_{A}} A_{\phi}$, where $\Psi_{A} \equiv\left\{\phi: \phi \in \Phi_{B} \cap A^{\prime}\right\}$ and $A_{\phi} \equiv\{x: x \in B, \phi(x) \geqq 0\}$.

Proof. Let $M \equiv\left\{\mu: \mu \in A^{\prime}, \mu(1)=1\right\}$. Then, because 0 is the only functional in $A^{\prime}$ which can vanish at the interior point $1, A^{\prime}=\left(\bigcup_{\lambda \geqq 0} \lambda M\right)$. Note that $\Psi_{A} \subseteq M$.

Suppose that $M$ is not void. Then $M$ is convex and weak* closed. For some positive $\delta, A$ contains the open $\delta$-ball with centre 1 , so that $M$ is contained in the closed $\delta^{-1}$-ball with centre 0 . Thus $M$ is weak* compact. The Krein-Milman Theorem $[8$, p. 78] asserts that $M$ possesses extreme points, of which it is the weak* closed convex hull. Let $\mu$ be one of these extreme points. If $u \in A,\|u\|<\delta$, $0<\mu(u)<1$ and $\mu_{1}(x) \equiv(\mu(u))^{-1} \mu(u x), \mu_{2}(x) \equiv(\mu(1-u))^{-1} \mu(x-u x)(\forall x \in B)$, then $1-u \in A, \mu_{1}, \mu_{2} \in M$ and $\mu=\mu(u) \mu_{1}+\mu(1-u) \mu_{2}$. Since $\mu$ is extreme, $\mu=\mu_{1}$ so that $\mu(u) \mu(x)=\mu(u x)(\forall x \in B)$. Evidently, this equation will hold for any element $u$ of $A$. Since $A$ has a nonvoid interior, $B=A-A$, so that $\mu$ is in fact a multiplicative functional, hence a member of $\Psi_{A}$. If $x \in \bigcap_{\phi \in \Psi_{A}} A_{\phi}$, then $\mu(x) \geqq 0$ for each functional $\mu$ in $A^{\prime}$, so that, since $A$ is closed, $x \in A$. (See $\left[8\right.$, p. 22].) On the other hand, for any $x \in A$, clearly $x \in \bigcap_{\phi \in \Psi_{A}} A_{\phi}$.

If $M$ is void, then $\Psi_{A}$ is void and $A^{\prime}=\{0\}$. If $A$ were proper in $B$, it would be possible by Theorem 6 of $\left[8\right.$, p. 22] to find a nontrivial functional in $A^{\prime}$. Hence, in this case also, $A=B=\bigcap_{\phi \in \Psi_{A}} A_{\phi}$.

Corollary. Any closed subsemi-algebra of $B$ whose interior contains 1 must contain $\bigcap_{\phi \in \Phi_{B}} A_{\phi}$. Thus $\bigcap_{\phi \in \Phi_{B}} A_{\phi}$ is the least closed semi-algebra containing 1 as interior point.

Proof. The first statement is clear. To see the second, observe that each $A_{\phi}$ contains the open unit ball with centre 1 , 
Definition. The principal semi-algebra $B_{+}$contained in $B$ is the least closed semi-algebra containing 1 as an interior point.

The corollary asserts that $B_{+}$certainly exists. One property of $B_{+}$which will be used later is that, for each element $u$ belonging to $G \cap B_{+}$, the inverse $u^{-1}$ also belongs to $B_{+}$.

\subsection{Maximal closed subsemi-algebras of $B_{+}$.}

Definition. A closed subsemi-algebra of $B_{+}$which is not properly contained in any proper closed subsemi-algebra of $B_{+}$is said to be maximal closed in $B_{+}$.

THEOREM 1.2. (i) If $\Phi_{B}$ is void or contains at least two elements, each maximal closed subsemi-algebra of $B_{+}$contains the identity 1 .

(ii) If $\Phi_{B}$ consists of the single element $\phi$, then $\left\{x: x \in B_{+}, \phi(x)=0\right\}$ is the unique maximal closed subsemi-algebra of $B_{+}$which does not contain 1 .

Before proving this theorem, we need some results about adjoining the identity to a subsemi-algebra $A$ of $B$. Let

$$
A_{1} \equiv\{a+\alpha: a \in A, \alpha \geqq 0\} .
$$

LEMMA 1.1. If $A$ is a closed subsemi-algebra of $B$, then $A_{1}$ is also a closed subsemi-algebra. Furthermore

$$
A_{1}^{\prime}=\left\{\mu: \mu \in A^{\prime}, \mu(1) \geqq 0\right\} .
$$

Proof. The result that $A_{1}$ is a semi-algebra and the equality for $A_{1}^{\prime}$ are straightforward. If $1 \in A$, then $A_{1}=A$ is closed. Suppose that 1 does not belong to $A$ and that $z$ lies in the closure of $A_{1}$. Then there exists a sequence $\left\{x_{n}+\alpha_{n}: x_{n} \in A, \alpha_{n} \in R^{+}\right\}$such that $\lim _{n \rightarrow \infty}\left(x_{n}+\alpha_{n}\right)=z$. If $\left\{\alpha_{n}\right\}$ has no convergent subsequence, then there is a subset $D$ of the positive integers such that $\lim \left\{\alpha_{n}: n \rightarrow \infty, n \in D\right\}=\infty$. Because $\left\|\alpha_{n}^{-1} x_{n}+1\right\| \leqq \alpha_{n}^{-1}\left\|x_{n}+\alpha_{n}-z\right\|+\alpha_{n}^{-1}\|z\|$, it follows that $-1=\lim \left\{\alpha_{n}^{-1} x_{n}: n \rightarrow \infty, n \in D\right\}$, so that $-1 \in A$. But then $1=(-1)^{2} \in A$, giving a contradiction. Hence $\left\{\alpha_{n}\right\}$ has a convergent subsequence with non-negative limit $\alpha$, say. It can be checked that $\left\{x_{n}\right\}$ has a corresponding subsequence with limit $z-\alpha$, which must lie in $A$. Thus $z=(z-\alpha)+\alpha \in A_{1}$, and $A_{1}$ is closed.

LEMMA 1.2. (i) If $\Phi_{B}$ is void, or contains at least two elements, then there is no proper closed subsemi-algebra $A$ of $B_{+}$with $A_{1}=B_{+}$.

(ii) If $\Phi_{B}$ consists of the single element $\phi$, then $A \equiv\{x: x \in B, \phi(x)=0\}$ is the unique closed subsemi-algebra of $B_{+}$with $A_{1}=B_{+}$.

Proof. By Theorem 1.1, if $\Phi_{B}$ is void, then $B_{+}=B$. If a closed semi-algebra $A$ satisfies $A_{1}=B_{+}=B$, then $-1=x+\alpha$ for some $x \in A$ and $\alpha \geqq 0$, with the result that $1=(1+\alpha)^{-2} x^{2} \in A$ and $A=B$. Now let $\Phi_{B}$ and $A$ be as specified in (ii) and let $x \in B_{+}$. Since $x=(x-\phi(x))+\phi(x), x$ belongs to $A_{1}$. 
If $\Phi_{B}$ is nonvoid, suppose that $A$ is a proper closed subsemi-algebra of $B_{+}$ with $A_{1}=B_{+}$. Then there exists a functional $\mu \in A^{\prime}$ with $\mu(1)=-1$. Let $\phi \in \Phi_{B}$. Then $\mu+\phi \in A^{\prime},(\mu+\phi)(1)=0$ so that $\mu+\phi$ is a functional in $B_{+}^{\prime}$ vanishing at the interior point 1 . Hence $\mu+\phi=0$, i.e. $\phi=-\mu$. Thus $\Phi_{B}$ consists of a single element and $\mu$ is the sole functional in $A^{\prime}$ with $\mu(1)=-1$. It follows that

$$
\begin{aligned}
& B_{+}^{\prime}=\left\{\lambda \phi: \lambda \in \boldsymbol{R}^{+}\right\}, \\
& A^{\prime}=\left\{\lambda \mu: \lambda \in \boldsymbol{R}^{+}\right\} \cup B_{+}^{\prime}=\{\lambda \phi: \lambda \in \boldsymbol{R}\},
\end{aligned}
$$

so that $A=\{x: x \in B, \phi(x)=0\}$.

Proof of Theorem 1.2. If $A$ is a maximal closed subsemi-algebra of $B_{+}$, either $1 \in A$ or, $A_{1}$ being closed, $A_{1}=B_{+}$. Lemma 1.2 shows that the only possibility for the latter case is that $\phi_{B}$ consists of a single element, $\Phi$ say, and that $A=\{x: x \in B, \phi(x)=0\}$. Suppose this to be so; then we show that, for each $z \in B_{+} \mid A$, the least closed semi-algebra $(A, z)$ containing $A$ and the element $z$ is in fact $B_{+}$, so that $A$ is indeed maximal.For, if $y \in B_{+}$, then $y-(\phi(y) / \phi(z)) z \in A$, so that $y=[y-(\phi(y) / \phi(z)) z]+[(\phi(y) / \phi(z)) z] \in(A, z)$. Then $B_{+} \subseteq(A, z)$, and the theorem follows.

REMARK. Case (ii) may actually occur; for example, $\{0\}$ is maximal closed in $\boldsymbol{R}^{+}$. See also $\$ 1.4$, Example 1.1.

THEOREM 1.3. Let $A$ be a proper closed subsemi-algebra of $B_{+}$which contains an interior point $a$. Then $A$ is contained in a maximal closed subsemialgebra of $B_{+}$.

Proof. It follows from Lemmata 1.1 and 1.2 that $A_{1}$ is also a proper closed subsemi-algebra of $B_{+}$. Choose $\lambda>0$ sufficiently large that $b=a+\lambda$ is regular. Since $\lambda \in A_{1}$ and $a \in \operatorname{int} A_{1}$, it follows that $b$ is an interior point of $A_{1}$. Choose an open neighborhood $U$ of $b$ which consists entirely of regular elements and is contained in $A_{1}$.

Let $P$ be any proper closed subsemi-algebra of $B_{+}$which contains $A_{1}$. Suppose, if possible, that $u \in U^{-1} \cap P$. Then $1=u u^{-1} \in u U \subseteq$ int $P$, so that $P=B_{+}$, contrary to assumption. Hence $U^{-1} \cap P=\varnothing$. Zorn's Lemma permits the choosing of a maximal chain (ordered by inclusion) of such semi-algebras $P$, and the closure of the union of the members of this chain is a maximal closed subsemialgebra of $B_{+}$containing $A_{1}$ (and hence $A$ ), but not intersecting the open set $U^{-1}$.

1.3. Geometric semi-algebras. In this section, suppose that $B$ is a real Banach algebra with $\Phi_{B} \neq \varnothing$.

Definition. Let $\sigma \in B^{*} . \sigma$ is a positive linear functional iff $\sigma(x) \geqq 0\left(\forall x \in B_{+}\right)$. $\sigma$ is a positive normalized linear functional (p.n.l.f.) iff $\sigma$ is positive and satisfies $\sigma(1)=1$. 
Suppose $\Phi_{B}$ to have the Gelfand topology. For $x \in B$, the function $f_{x}$ defined on $\Phi_{B}$ by

$$
f_{x}(\phi)=\phi(x) \quad\left(\forall \phi \in \Phi_{B}\right)
$$

is continuous. The mapping $x \rightarrow f_{x}$ is a continuous homomorphism of $B$ onto a uniformly dense subalgebra $C_{B}$ of $C\left(\Phi_{B}\right)$. For a p.n.l.f. $\sigma$ on $B$, define the functional $\bar{\sigma}$ on $C_{B}$ by

$$
\bar{\sigma}\left(f_{x}\right)=\sigma(x) \quad(\forall x \in B) .
$$

It is easily verified that, if $f_{x}=f_{y}$, then $\sigma(x)=\sigma(y)$, so that the definition is meaningful. The positivity of $\bar{\sigma}$ on $C_{B} \cap C^{+}\left(\Phi_{B}\right)$ means that $\bar{\sigma}$ can be uniquely extended to a Radon probability measure defined on the whole of $C\left(\Phi_{B}\right)$.

Definition. Let $\sigma$ be a p.n.l.f. on $B$; denote by $\bar{\sigma}$ the probability measure induced by $\sigma$ on $\Phi_{B}$. The functional $\gamma_{\sigma}$ is defined for $x \in B_{+}$by

$$
\gamma_{\sigma}(x)=G M_{\bar{\sigma}} f_{x} .
$$

LEMMA 1.3. Let $\sigma$ be a p.n.l.f. on $B$. For $x, y \in B_{+}, \lambda \geqq 0$ :

(a) $0 \leqq \gamma_{\sigma}(x) ; \gamma_{\sigma}(\lambda)=\lambda$;

(b) $y-x \in B_{+} \Rightarrow \gamma_{\sigma}(x) \leqq \gamma_{\sigma}(y)$;

(c) $\lim _{\lambda \rightarrow 0}+\gamma_{\sigma}(x+\lambda y)=\gamma_{\sigma}(x)$;

(d) $0 \leqq \gamma_{\sigma}(x) \leqq \sigma(x) \leqq\|x\|$;

(e) $\gamma_{\sigma}(x y)=\gamma_{\sigma}(x) \gamma_{\sigma}(y)$;

(f) $\gamma_{\sigma}(x+y) \geqq \gamma_{\sigma}(x)+\gamma_{\sigma}(y)$;

(g) $\gamma_{\sigma}(\lambda x)=\lambda \gamma_{\sigma}(x)$

(h) if $x \in G$, then $\gamma_{\sigma}\left(x^{-1}\right)=\left(\gamma_{\sigma}(x)\right)^{-1}$.

For $\phi \in \Phi_{B}$, it is true that $\gamma_{\phi}(x)=\phi(x)$.

Let $\sigma_{1}$ and $\sigma_{2}$ be two p.n.l.f.'s on $B$ and suppose that $\sigma=\alpha \sigma_{1}+(1-\alpha) \sigma_{2}$ $(0<\alpha<1)$. Then $\gamma_{\sigma}(x)=\left(\gamma_{\sigma_{1}}(x)\right)^{\alpha}\left(\gamma_{\sigma_{2}}(x)\right)^{1-\alpha}$ for all $x \in B_{+}$.

Proof. These properties are consequences of the corresponding properties of $G M_{\bar{\sigma}}$ given in $\S 0.2$ and the homomorphic character of the mapping $x \rightarrow f_{x}$. For example, (b) is proved by the following chain of implications:

$$
\begin{aligned}
y-x \in B_{+} & \Rightarrow\left(f_{y}-f_{x}\right)(\phi)=\left(f_{y-x}\right)(\phi) \geqq 0 \quad\left(\forall \phi \in \Phi_{B}\right) \\
& \Rightarrow f_{y} \geqq f_{x} \Rightarrow \gamma_{\sigma}(y)=G M_{\bar{\sigma}} f_{y} \geqq G M_{\bar{\sigma}} f_{x}=\gamma_{\sigma}(x) .
\end{aligned}
$$

LEMMA 1.4. Let $\sigma$ be a p.n.l.f. on $B$. Then $\gamma_{\sigma}$ is continuous on the interior of $B_{+}$; in particular, $\gamma_{\sigma}$ is continuous on $G \cap B_{+}$.

Proof. Note that $x \rightarrow \gamma_{\sigma}(x)$ is the composition of the continuous mappings $x \rightarrow f_{x}$ (of int $B_{+}$into $C^{*}\left(\Phi_{B}\right)$ ), $f \rightarrow \log f$ (of $C^{*}\left(\Phi_{B}\right)$ into $C\left(\Phi_{B}\right)$ ), $f \rightarrow \bar{\sigma}(f)$ (of $C\left(\Phi_{B}\right.$ ) into $R$ ), $t \rightarrow e^{t}$ (of $R$ into $R^{+}$). 
THeOREM 1.4. Let $\sigma$ be a p.n.l.f. on $B$ and let $\psi \in \Phi_{B}$. Define:

$$
H_{\sigma, \psi} \equiv\left\{x: x \in B_{+}, \psi(x) \leqq \gamma_{\sigma}(x)\right\} .
$$

If $\sigma=\psi$, then $H_{\sigma, \psi}=B_{+}$. If $\sigma \neq \psi$, then $H_{\sigma, \psi}$ is a proper closed subsemi-algebra of $B_{+}$which contains the identity.

Proof. If $\sigma=\psi$, then $\gamma_{\sigma}=\psi$, by Lemma 1.3, so that the first statement is true. Henceforth, assume that $\sigma \neq \psi$. If $H_{\sigma, \psi}$ were not proper in this case, then $\psi(x) \leqq \gamma_{\sigma}(x)$ for each $x \in B_{+}$, whereupon $(\log f)(\psi) \leqq \bar{\sigma}(\log f)$ for each function $f$ in $C^{\#}\left(\Phi_{B}\right) \subseteq \operatorname{Cl}\left(C_{B} \cap C^{*}\left(\Phi_{B}\right)\right)$, and $f(\psi) \leqq \bar{\sigma}(f)$ for each function $f$ in $C\left(\Phi_{B}\right)$. Since $C\left(\Phi_{B}\right)$ is closed under the taking of additive inverses, it follows that $f(\psi)=\bar{\sigma}(f)$ $\left(\forall f \in C\left(\Phi_{B}\right)\right)$, and a contradiction is obtained.

The properties of the functional $\gamma_{\sigma}$ listed in Lemma 1.3 can be used to show that $H_{\sigma, \psi}$ is a semi-algebra containing the identity. Suppose that $y$ belongs to the closure of $H_{\sigma, \psi}$. Then there exists a sequence $\left\{y_{n}\right\}$ such that $y_{n} \in H_{\sigma, \psi}$ and $\left\|y-y_{n}\right\|<n^{-1}$ for each positive integer $n$. Therefore $\left|\phi\left(y-y_{n}\right)\right|<n^{-1}$, so that

$$
\phi(y-(1 / n))<\phi\left(y_{n}\right)<\phi(y+(1 / n))
$$

for each $\phi \in \Phi_{B}$, each positive integer $n$. Since $\phi\left(y+(1 / n)-y_{n}\right)>0\left(\forall \phi \in \Phi_{B}\right)$, it is true that $y+(1 / n)-y_{n} \in B_{+}$, so that, by Lemma 1.3(b), $\gamma_{\sigma}\left(y_{n}\right) \leqq \gamma_{\sigma}(y+(1 / n))$. Hence:

$$
\psi(y-(1 / n)) \leqq \psi\left(y_{n}\right) \leqq \gamma_{\sigma}\left(y_{n}\right) \leqq \gamma_{\sigma}(y+(1 / n))
$$

for each positive integer $n$. Letting $n$ tend to infinity and making use of Lemma 1.3(c), we obtain that $\psi(y) \leqq \gamma_{\sigma}(y)$, so that $y \in H_{\sigma, \psi}$. Thus, $H_{\sigma, \psi}$ is closed and the proof is complete.

DEFINITION. A geometric semi-algebra contained in $B_{+}$is a semi-algebra of the form $H_{\sigma, \psi}$ where $\sigma$ is a p.n.l.f. on $B$ and $\psi \in \Phi_{B}$. It is implicit in the definition that $\sigma \neq \psi$.

For any geometric semi-algebra, it can always be arranged that the p.n.l.f. involved in the definition is not a nontrivial convex combination of the homomorphism involved and another p.n.l.f. For, if $\sigma=\alpha \psi+(1-\alpha) \omega$ where $0<\alpha<1$ and $\omega$ is a p.n.l.f., then, by the final part of Lemma $1.3, H_{\sigma, \psi}=H_{\omega, \psi}$. The remainder of this section will be devoted to showing that the geometric semi-algebras are maximal closed subsemi-algebras of the principal semi-algebra. For $H$ a closed subsemi-algebra of $B_{+}$and $v$ an element of $B_{+}$, the least closed subsemi-algebra of $B_{+}$containing $H$ and the element $v$ will be denoted by $(H, v)$. The maximality of $H$ is equivalent to the property that $(H, v)=B_{+}$whenever $v \in B_{+} \backslash H$.

LEMMA 1.5. Let $H_{\sigma, \psi}$ be a geometric semi-algebra contained in $B_{+}$. If $u$ is an element of $B_{+}$belonging to $G \backslash H_{\sigma, \psi}$, then $\left(H_{\sigma, \psi}, u\right)=B_{+}$. 
Proof. It can be seen that $\gamma_{\sigma}\left(u^{-1}\right)>\psi\left(u^{-1}\right)$, so that by the continuity of $\gamma_{\sigma}$ and $\psi$ on $B_{+} \cap G$, there exists a neighbourhood $V$ of $u^{-1}$ contained in $H_{\sigma, \psi}$. Then $u V$ is a neighbourhood of 1 contained in $\left(H_{\sigma, \psi}, u\right)$, and the result follows by the definition of $B_{+}$.

LEMMA 1.6. Let $H_{\sigma, \psi}$ be a geometric semi-algebra contained in $B_{+}$. If $v \in B_{+}$and $\psi(v)>\sigma(v)$, then $\left(H_{\sigma, \psi}, v\right)=B_{+}$.

Proof. Choose $\lambda \in(0,1]$ such that $\lambda<(\|1-v\|)^{-1}$. Then $u=\lambda v+(1-\lambda)$ satisfies the conditions of Lemma 1.5. Hence $B_{+}=\left(H_{\sigma, \psi}, u\right) \subseteq\left(H_{\sigma, \psi}, v\right) \subseteq B_{+}$.

LEMMA 1.7. Let $H_{\sigma, \psi}$ be a geometric semi-algebra contained in $B_{+}$. If $v \in B_{+} \backslash H_{\sigma, \psi}$, and if $\psi(v) \leqq \sigma(v)$, then $\left(H_{\sigma, \psi}, v\right)=B_{+}$.

Proof. Choose a positive real $\lambda$ sufficiently small that $u=v+\lambda$ does not belong to $H_{\sigma, \psi}$. Then $f_{u}$ is a regular element of $C^{+}\left(\Phi_{B}\right)$ and $\left(f_{u}\right)(\psi)=\psi(u)>\gamma_{\sigma}(u)$ $=G M_{\bar{\sigma}} f_{u}$, so that $\left(f_{u}\right)^{-1}(\psi)<G M_{\bar{\sigma}}\left(f_{u}\right)^{-1}$ for the multiplicative inverse of $f_{u}$. Choose a uniform neighbourhood $V$ of $f_{u}^{-1}$ with $V \subseteq C^{*}\left(\Phi_{B}\right)$ and $f(\psi)<G M_{\bar{\sigma}} f$ $(\forall f \in V)$. Since $C_{B} \cap C^{+}\left(\Phi_{B}\right)$ is uniformly dense in $C^{+}\left(\Phi_{B}\right)$, it is possible to find in $V$ a function $f_{z}$, for some $z \in B_{+}$, such that $\left(f_{u} f_{z}\right)(\psi)-\bar{\sigma}\left(f_{u} f_{z}\right)>0$. (Note that $f_{u}^{-1}$ lies in the proper closed hyperplane $\left\{f: f \in C\left(\Phi_{B}\right),\left(f_{u} f\right)(\psi)-\bar{\sigma}\left(f_{u} f\right)=0\right\}$.) Then $z \in H_{\sigma, \psi}$ and $\psi(u z)>\sigma(u z)$. Hence, $u z \in\left(H_{\sigma, \psi}, u\right)$ and, by Lemma 1.6,

$$
B_{+}=\left(H_{\sigma, \psi}, u z\right) \subseteq\left(H_{\sigma, \psi}, u\right) \subseteq\left(H_{\sigma, \psi}, v\right) \subseteq B_{+} .
$$

Combining Lemmata 1.6 and 1.7, and noting that for distinct elements $\psi_{1}$ and $\psi_{2}$ in $\Phi_{B}, H_{\psi_{1}, \psi_{2}}$ is a geometric semi-algebra, we obtain the following result:

THEOREM 1.5. Let $B$ be a real Banach algebra with identity such that $\Phi_{B}$ possesses at least two elements. Then $B_{+}$contains geometric semi-algebras and each such semi-algebra is a maximal closed subsemi-algebra of $B_{+}$.

REMARK. If $\Phi_{B}$ has a single element, then this element is the only p.n.l.f.

1.4. Other examples of maximal closed subsemi-algebras. In general, not every maximal closed subsemi-algebra of the principal semi-algebra of a Banach algebra is geometric. This section indicates other possibilities.

THEOREM 1.6. Let $\psi$ be a complex homomorphism on $B$ not contained in $\Phi_{B}$. Then

$$
K_{\psi} \equiv\left\{x: x \in B_{+}, \psi(x) \text { is real }\right\}
$$

is a maximal closed subsemi-algebra of $B_{+}$.

Proof. Evidently $K_{\psi}$ is a proper closed subsemi-algebra of $B_{+}$which contains the identity. For $u \in B_{+} \backslash K_{\psi}$, let $\left(K_{\psi}, u\right)$ be the least closed semi-algebra 
containing $K_{\psi}$ and $u$, and suppose that $\psi(u)=\lambda e^{i \theta}$. Note that $\theta$ is not an integral multiple of $\pi$.

Suppose $v \in B_{+}$and that $\psi(v)=\alpha e^{i \omega}$. If $\omega$ is an integral multiple of $\pi$, then $v \in K_{\psi}$. If $\omega$ is not an integral multiple of $\pi$, then positive real $\beta$ and $\rho$, and a positive integer $m$ can be chosen so that $\lambda e^{i \theta}+\beta=\rho e^{i \omega / m}$ (for $\omega$ suitably modified if necessary) and $u+\beta$ is regular. It can be checked that $u+\beta \in\left(K_{\psi}, u\right)$ and that $v(u+\beta)^{-m} \in K_{\psi} \subseteq\left(K_{\psi}, u\right)$. Hence $v=v(u+\beta)^{-m}(u+\beta)^{m} \in\left(K_{\psi}, u\right)$. The required result follows.

RemarK. A semi-algebra of the form $K_{\psi}$ may certainly be strict (i.e. $x,-x \in K_{\psi} \Rightarrow x=0$ ). For, if $B$ be the real Banach algebra of functions continuous on the closed unit disc, analytic on its interior and taking real values on the real axis, then $B_{+}$, and, a fortiori, the $K_{\psi}$ (corresponding to points of the disc not on the real axis) are strict.

The sort of situation which may occur even when there are no strictly complex homomorphisms on $B$ is illustrated by two examples.

EXAMPLE 1.1. Let $K^{(2)}$ be the set of all real triples $x \equiv\left(x_{0}, x_{1}, x_{2}\right)$ with the norm and algebraic operations defined by:

$$
\begin{aligned}
\|x\| & =\sum_{i=0}^{2}\left|x_{i}\right|, \\
(x+y)_{i} & =x_{i}+y_{i}, \\
(x y)_{i} & =\sum_{j=0}^{i} x_{j} y_{i-j}, \quad i=0,1,2 \\
(\lambda x)_{i} & =\lambda x_{i}
\end{aligned}
$$

for elements $x$ and $y$, and real $\lambda$. The multiplicative identity is the element $1 \equiv(1,0,0)$. Since $\Phi_{K^{(2)}}$ consists of the single homomorphism $x \rightarrow x_{0}$, the princinal semi-algebra $K_{+}^{(2)}$ is the set $\left\{x: x \in K^{(2)}, x_{0} \geqq 0\right\}$.

Define the sets:

$$
\begin{aligned}
& P \equiv\left\{x: x \in K_{+}^{(2)}, x_{1} \geqq 0\right\}, \\
& Q \equiv\left\{x: x \in K_{+}^{(2)}, x_{1} \leqq 0\right\}, \\
& R \equiv\left\{x: x \in K_{+}^{(2)}, x_{0}=0\right\}, \\
& S_{\beta} \equiv\left\{x: x \in K_{+}^{(2)} ; 2 x_{0} x_{2}-x_{1}^{2} \geqq \beta x_{0} x_{1} ; x_{1}=0, x_{2} \geqq 0 \text { when } x_{0}=0\right\},
\end{aligned}
$$

where $\beta$ is a real number. Each of these sets is a maximal closed subsemi-algebra of $K_{+}^{(2)}$. The maximality of $R$ is a consequence of Theorem 1.2. The maximality of the others can be proved by an argument similar to that used for geometric semi-algebras.

EXAMPLE 1.2. Let $C^{(2)}[0,1]$ be the Banach algebra of all real-valued continuous twice continuously differentiable functions defined on the closed unit 
interval, with the algebraic operations defined pointwise and the norm $\|\cdot\|$ given by $\|f\| \equiv \sup \left\{|f(t)|+\left|f^{\prime}(t)\right|+\frac{1}{2}\left|f^{\prime \prime}(t)\right|: 0 \leqq t \leqq 1\right\}$ for any member $f$. The principal semi-algebra $C_{+}^{(2)}[0,1]$ is precisely the set of functions which take only non-negative values.

For $s$ a fixed point of $[0,1], \beta$ some real number and $\alpha$ some nonpositive real number, define the linear functional $\rho$ on $C^{(2)}[0,1]$ by

$$
\rho(g) \equiv g(s)+\beta g^{\prime}(s)+\alpha g^{\prime \prime}(s) \quad\left(\forall g \in C^{(2)}[0,1]\right) .
$$

The functional $\rho$ can be extended in an obvious manner so that when $f \in C_{+}^{(2)}[0,1]$ and $f(s)>0, \log f$ exists in a neighbourhood of $s$ and $\rho(\log f)$ is defined. Define $\tau_{\rho}$ for $f \in C_{+}^{(2)}[0,1]$ by

$$
\tau_{\rho}(f) \equiv \begin{cases}\exp \rho(\log f) & \text { whenever } f(s)>0 \\ \lim _{\lambda \rightarrow 0}+\tau_{\rho}(f+\lambda) & \text { whenever } f(s)=0 .\end{cases}
$$

$\tau_{\rho}$ may be positively infinite when $f(s)=0$. Leaving the products $\infty \cdot \infty$ and $0 \cdot \infty$ undefined and using the usual conventions otherwise in dealing with infinity, we can show that, for $f, g \in C_{+}^{(2)}[0,1]$ :

$$
\begin{aligned}
0 & \leqq \tau_{\rho}(f) ; \tau_{\rho}(f+g) \leqq \tau_{\rho}(f)+\tau_{\rho}(g) ; \\
\tau_{\rho}(\lambda) & =\lambda, \tau_{\rho}(\lambda f)=\lambda \tau_{\rho}(f)(\forall \lambda \geqq 0) ; \\
\tau_{\rho}(f g) & =\tau_{\rho}(f) \tau_{\rho}(g),
\end{aligned}
$$

whenever the latter product is defined. For $\sigma$ a p.n.l.f. on $C^{(2)}[0,1]$ not equal to $\rho$, define the set

$$
H_{\sigma, \rho} \equiv\left\{f \in C_{+}^{(2)}[0,1], \quad \tau_{\rho}(f) \leqq \gamma_{\sigma}(f)\right\} .
$$

$H_{\sigma, \rho}$ is a maximal closed subsemi-algebra of $C_{+}^{(2)}[0,1]$.

\section{CHAPTER 2}

2.1. Intersections of geometric semi-algebras contained in $C^{+}(E)$. In this section a characterization of those subsemi-algebras of $C^{+}(E)$ which are the intersections of families of geometric semi-algebras will be given. Define:

$$
H_{\sigma, \xi} \equiv\left\{f: f \in C^{+}(E), f(\xi) \leqq G M_{\sigma} f\right\}
$$

for a point $\xi$ in $E$ and a probability measure $\sigma$ on $E$. Since every homomorphism of $C(E)$ into the real numbers arises from a point of $E$, each geometric semialgebra has the form $H_{\sigma, \xi}$.

Definition. A subset $S$ of $C^{+}(E)$ is power closed iff, for each positive real $\lambda$, $S$ contains, along with any member $f$, the function $f^{\lambda}$, defined for $\xi \in E$ by

$$
f^{\lambda}(\xi) \equiv \text { principal value }(f(\xi))^{\lambda} \text {. }
$$

A uniformly closed power closed subsemi-algebra of $C^{+}(E)$ is called a cornet. 
Any geometric semi-algebra is a cornet with identity. Further, since for $f$, $g \in C^{+}(E), \lim _{n \rightarrow \infty}\left(f^{n}+g^{n}\right)^{1 / n}=f \cup g$, each cornet is an upper semi-lattice. (The term "lim" refers to the taking of the uniform limit.)

THEOREM 2.1. Let $P$ be a uniformly closed subset of $C^{+}(E)$ which satisfies the conditions:

(i) $P$ is closed under multiplication;

(ii) $\lambda P \subseteq P$ for each positive real $\lambda$;

(iii) $P$ is an upper semi-lattice;

(iv) $P$ is power closed;

(v) $P$ contains the identity function 1 .

Let $\mathscr{F}$ be the set of all pairs $(\sigma, \xi)$ with $\sigma$ a probability measure and $\xi$ a point of $E$, such that $P \subseteq H_{\sigma, \xi}$. Then:

$$
P=\bigcap_{(\sigma, \xi)} H_{\sigma, \xi} \cdot
$$

Remark. It is not stipulated that $P$ is closed under addition. This more general form of the theorem will be useful later. The theorem is valid even when $\mathscr{F}$ is void.

Proof. Let $P^{*}=P \cap C^{*}(E)$. Then, since $f \cup \varepsilon \in P^{*}$ for $f \in P$ and $\varepsilon>0$, $P$ is the uniform closure of $P^{*}$. The set $W \equiv \log P^{*}$ is a closed wedge in $C(E)$ which is an upper semi-lattice. Let $\mathscr{U}_{1}$ and $\mathscr{U}_{2}$ be defined with respect to $W$ as in Theorem 0.1. Because the functions 1 and -1 belong to $W, \mathscr{U}_{2}$ consists of the zero measure alone and, for each $(\sigma, \xi) \in \mathscr{U}_{1}, \sigma$ is a probability measure.

By Theorem $0.1, W=\bigcap_{(\sigma, \xi) \in \mathscr{U}_{1}} U_{\sigma, \xi}$, so that $P^{*}=\left(\bigcap_{(\sigma, \xi) \in \mathscr{U}_{1}} H_{\sigma, \xi}\right) \bigcap^{*}(E)$. It is straightforward to show that $P=\bigcap_{(\sigma, \xi) \in \mathscr{U}_{1}} H_{\sigma, \xi}$. Finally, noting that $\mathscr{U}_{1} \subseteq \mathscr{F}$ and that $P \subseteq H_{\sigma, \xi}$ for $(\sigma, \xi) \in \mathscr{F}$, we obtain that $P=\bigcap_{(\sigma, \xi) \in \mathscr{U}_{1}} H_{\sigma, \xi}$ $\supseteq \bigcap_{(\sigma, \xi) \in \mathscr{F}} H_{\sigma, \xi} \supseteq P$.

COROLlaRY 1. The set $P$ described in the theorem is a semi-algebra (and hence a cornet).

COROLlaRy 2. Let $A$ be a cornet with identity, $\mathscr{F}$ be the set of all pairs $(\sigma, \xi)$ with $\sigma$ a probability measure, $\xi$ a point of $E$, such that $A \subseteq H_{\sigma, \xi}$. Then $A=\bigcap_{(\sigma, \xi) \in \mathscr{F}} H_{\sigma, \xi}$.

The next result is a useful application of Theorem 2.1. For a subsemi-algebra $A$ of $C(E)$, define the subset of $E$ :

$$
N(A) \equiv\{\eta: \eta \in E, f(\eta)=0 \quad(\forall f \in A)\} .
$$

THEOREM 2.2. Let $A$ be a uniformly closed subsemi-algebra of $C^{+}(E)$ with $N(A)$ void. Define:

$$
\begin{aligned}
& P_{A} \equiv\left\{f: f \in C^{+}(E), f^{n} \in A \text { for some positive integer } n \equiv n(f)\right\} \\
& \sqrt{ } A \equiv \bar{P}_{A}, \text { the uniform closure of } P_{A} . \\
& \text { Then } \sqrt{ } A \text { is the least cornet containing } A \text {. }
\end{aligned}
$$


Proof. Clearly, any cornet containing $A$ must contain $\sqrt{ } A$. It remains only to show that $\sqrt{ } A$ is a cornet. We show that $\sqrt{ } A$ satisfies the five conditions of Theorem 2.1 and apply Corollary 1 .

(i) Evidently, $P_{\boldsymbol{A}}$ is closed under multiplication; hence, the closure of $P_{\boldsymbol{A}}$ is also.

(ii) $P_{A}$, and hence its closure, is closed under multiplication by non-negative scalars.

(iii) Let $f, g \in P_{A}$. Choose positive integers $m, n$ such that $f^{m}$ and $g^{n}$ belong to $A$. Then for every positive integer $k, f^{m n k}+g^{m n k} \in A$, so that $\left(f^{m n k}+g^{m n k}\right)^{1 / m n k} \in P_{A}$. Then (letting $k$ tend to infinity) we see that $f \cup g \in \sqrt{ } A$. If $f$ and $g$ are functions in $\sqrt{ } A$, then there exist sequences $\left\{f_{n}\right\}$ and $\left\{g_{n}\right\}$ contained in $P_{A}$ with limits $f$ and $g$ respectively. Since, for each positive integer $n, f_{n} \cup g_{n} \in \sqrt{ } A$, then $f \cup g=\lim _{n \rightarrow \infty}\left(f_{n} \cup g_{n}\right) \in \sqrt{ } A$.

(iv) If $f \in P_{A}$, then for any pair $p, q$ of positive integers $f^{p / q} \in P_{A}$. Then, for each positive real $\lambda, f^{\lambda} \in \sqrt{ } A$. If $f \in \sqrt{ } A, f^{\lambda}$ can be approximated by a sequence $\left\{f_{n}^{\lambda}: f_{n} \in P_{A}\right\}$, so that $f^{\lambda} \in \sqrt{ } A$.

(v) For each point $\xi$ in $E$, there exists a function $f_{\xi} \in A$ such that $f_{\xi}$ is positive on a neighbourhood of $\xi$. Because of the compactness of $E$, it is possible to find a finite set of such functions $f_{\xi}$ whose sum $f$ is an everywhere positive function belonging to $A$. Then $1=\lim _{\lambda \rightarrow 0}+f^{\lambda} \in \sqrt{ } A$.

The theorem now follows.

So far, the theorems of this section have given information only about cornets which contain the identity. Let $A$ now be an arbitrary cornet, and denote by $A_{c}$ the least cornet which contains $A$ along with the identity function 1 . Observe that $A=A_{c}$ if and only if $1 \in A$, and if and only if the set $N(A)$ is void. Note also that $A_{c}$ will not in general be the same as the semi-algebra $A_{1}$ defined in $§ 1.2$. The investigation of cornets without the identity involves the following lemma.

LEMMA 2.1. Let $f, g$ be two functions in $C^{+}(E)$ and let $\beta, \varepsilon$ be positive real numbers. Suppose that for some point $\eta$ in $E, f(\eta)=0$, and that for some positive integer $n,\left\|f-(g+\beta)^{1 / n}\right\|<\frac{1}{2} \varepsilon$. Then $\left\|f-g^{1 / n}\right\|<\varepsilon$.

Proof. Clearly $0<\beta \leqq g(\eta)+\beta<\left(\frac{1}{2} \varepsilon\right)^{n}$. Since $(t+\beta)^{1 / n}-t^{1 / n}$ is decreasing for non-negative values of $t$, it follows that for each point $\xi$ in $E$,

$$
0 \leqq(g+\beta)^{1 / n}(\xi)-g^{1 / n}(\xi) \leqq \beta^{1 / n}<\frac{1}{2} \varepsilon
$$

Thus,

$$
\left\|f-g^{1 / n}\right\| \leqq\left\|f-(g+\beta)^{1 / n}\right\|+\left\|(g+\beta)^{1 / n}-g^{1 / n}\right\|<\varepsilon .
$$

There is a very simple relationship between the cornets $A$ and $A_{c}$. In fact, we show that

$$
A=\left\{f: f \in A_{c}, f(\eta)=0(\forall \eta \in N(A))\right\}
$$


This is true if $N(A)=\varnothing$. Suppose henceforth that $N(A) \neq \varnothing$. Evidently, $A$ is contained in the right-hand side. Since $A_{c}$ is the least cornet containing the closed semi-algebra $A_{1}$, it follows from Theorem 2.2 that $A_{c}=\sqrt{ } A_{1}$. Suppose that the function $f$ belongs to $A_{c}$ and vanishes on $N(A)$. For given positive $\varepsilon$, there exists a function $g \in A$, a positive real $\beta$ and a positive integer $n$ such that $\left\|f-(g+\beta)^{1 / n}\right\|<\frac{1}{2} \varepsilon$. Since $f$ vanishes on the nonvoid set $N(A)$, Lemma 2.1 shows that $\left\|f-g^{1 / n}\right\|<\varepsilon$. Because $A$ is a cornet containing $g, A$ contains $g^{1 / n}$ : let $f_{\varepsilon}=g^{1 / n}$. It is concluded that for any function $f$ in $A_{c}$ vanishing on $N(A)$, there exists a function $f_{\varepsilon}$ in $A$ with $\left\|f_{\varepsilon}-f\right\|<\varepsilon$; hence $f \in A$.

The connection between $A$ and $A_{c}$ leads directly to the following result.

THEOREM 2.3. Let $A$ be a cornet. Suppose that $\mathscr{F}$ is the set of pairs $(\sigma, \xi)$ with $\sigma$ a probability measure on $E$ and $\xi$ a point of $E$, such that $A \subseteq H_{\sigma, \xi}$. Then

$$
A=\left\{f: f \in H_{\sigma, \xi}(\forall(\sigma, \xi) \in \mathscr{F}), f(\eta)=0(\forall \eta \in N(A))\right\} .
$$

THEOREM 2.4. Let $A$ be a uniformly closed subsemi-algebra of $C^{+}(E)$. Define $\sqrt{ } A$ exactly as in Theorem 2.2. Then $\sqrt{ } A$ is the least cornet containing $A$.

Proof. Only the case $N(A) \neq \varnothing$ is to be considered. The arguments used in Theorem 2.2 apply here to show that $\sqrt{ } A$ is closed under multiplication, $\lambda \sqrt{ } A \subseteq \sqrt{ } A$ and $\sqrt{ } A$ is power closed. It remains to be verified that $\sqrt{ } A$ is closed under addition.

$\sqrt{ } A$ is contained in $\sqrt{ } A_{1}$, which, by Theorem 2.2 , is known to be a semi-algebra. Therefore, if $f, g \in \sqrt{ } A$, then $f+g \in \sqrt{ } A_{1}$. By the definition of $\sqrt{ } A_{1}$, for given positive $\varepsilon$, there exists $h \in A, \alpha>0$, a positive integer $n$, with $\left\|(f+g)-(h+\alpha)^{1 / n}\right\|<\frac{1}{2} \varepsilon$. Since $N(A)=N(\sqrt{ } A), f, g$ and $f+g$ all vanish on $N(A)$. By Lemma 2.1, $\left\|(f+g)-h^{1 / n}\right\|<\varepsilon$. Thus, $f+g$ can be uniformly approximated by integral roots of elements of $A$, so that $f+g \in \sqrt{ } A$. Hence $\sqrt{ } A$ is a cornet, and the theorem follows.

2.2. Every maximal closed subsemi-algebra of $C^{+}(E)$ is geometric. Let $\mu$ and $v$ be two Radon measures on $E$. Following F. Riesz (see [14] or [3]), define for $f \in C^{+}(E)$ the functional

$$
\sigma(f) \equiv \sup \left\{\mu(g)+v(f-g): 0 \leqq g \leqq f, g \in C^{+}(E)\right\} .
$$

This functional is linear and continuous on $C^{+}(E)$, and can be extended uniquely to a Radon measure, which is denoted by $\mu \cup v$. Define: $\mu_{+} \equiv \mu \cup 0, \mu_{-} \equiv(-\mu) \cup 0$. Then $\mu_{+}$and $\mu_{-}$are positive Radon measures and $\mu=\mu_{+}-\mu_{-}$. For $f \in C(E)$, the Radon measure $f \cdot \mu$ is defined by $(f \cdot \mu)(g) \equiv \mu(f g)(\forall g \in C(E))$.

If $K_{1}$ and $K_{2}$ are two subcones of $C^{+}(E)$ and $\mu_{1} \in K_{1}^{\prime}, \mu_{2} \in K_{2}^{\prime}$, then $\mu_{1} \cup \mu_{2} \in\left(\mathrm{Cl}\left(K_{1}+K_{2}\right)\right)^{\prime}$.

LEMMA 2.2. Let $f$ be a function in $C^{+}(E)$, and $\delta$ a real number such that $0<\delta<f(\xi)<1(\forall \xi \in E)$. Suppose that $\mu$ is a Radon measure on $E$ 
Then $(f \cdot \mu) \cup \mu$ is a positive measure if and only if $\mu$ is a positive measure.

Proof. The positivity of $\mu$ implies that of $f \cdot \mu$, and hence that of $(f \cdot \mu) \cup \mu$. Suppose that $\mu$ is not positive. Then $\mu_{-}$is nontrivial, so that a function $h \in C^{+}(E)$ can be found to satisfy

$$
0<\mu(-h) \leqq \mu_{-}(h) \leqq(1-\delta)^{-1} \mu(-h) .
$$

Then

$$
\begin{aligned}
((f \cdot \mu) \cup \mu)(h) & =\sup \{(f \cdot \mu)(g)+\mu(h-g): 0 \leqq g \leqq h\} \\
& =\mu(h)+\sup \{\mu(-(1-f) g): 0 \leqq g \leqq h\} \\
& \leqq-(1-\delta) \mu_{-}(h)+\mu_{-}((1-f) h) \\
& =\mu_{-}((\delta-f) h)<0,
\end{aligned}
$$

so that $(f \cdot \mu) \cup \mu$ is not positive.

LEMMA 2.3. Let $A$ be a proper uniformly closed subsemi-algebra of $C^{+}(E)$. Suppose the function $f$ in $C^{+}(E)$ is such that

(i) for some real $\delta, 0<\delta<f(\xi)<1(\forall \xi \in E)$;

(ii) $f^{2}$ belongs to $A$.

Then $(A, f)$, the least closed semi-algebra containing $A$ and the function $f$ is also proper in $C^{+}(E)$.

Proof. If $f \in A$, the result is obvious. Suppose then that $f \notin A$. Since $A$ is proper, there exists a nonpositive Radon measure $\mu$ belonging to $A^{\prime}$. Then $f \cdot \mu$ belongs to $(A f)^{\prime}$. Hence $(f \cdot \mu) \cup \mu$ is a nonpositive Radon measure in $(\mathrm{Cl}(A+A f))^{\prime}$, so that the cone $\mathrm{Cl}(A+A f)$ is a proper cone of $C^{+}(E)$. Now $A+A f$ is a semi-algebra contained in every closed semi-algebra containing $A$ and the function $f$. Hence $\operatorname{Cl}(A+A f)=(A, f)$ and the lemma follows.

THEOREM 2.5. Let $A$ be a maximal closed subsemi-algebra of $C^{+}(E)$. Then $A$ is power closed. If $E$ contains at least two distinct points, then each maximal closed subsemi-algebra of $\mathrm{C}^{+}(E)$ is a geometric semi-algebra.

Proof. If $E$ consists of a single point, then $C^{+}(E)$ is isomorphic to the semialgebra of non-negative reals, so that the only proper closed subsemi-algebra of $C^{+}(E)$ is $\{0\}$, and this is power closed.

If $E$ consists of more than one point, then the maximality of $A$ implies, by Theorem 1.2, that $1 \in A$. Let $g$ be an element of $A$ which is bounded away from zero. Define

$$
f \equiv\left(2\left\|g^{1 / 2}\right\|\right)^{-1} g^{1 / 2} ; \delta \equiv \inf \left\{\left(3\left\|g^{1 / 2}\right\|\right)^{-1} g^{1 / 2}(\xi): \xi \in E\right\} .
$$

Then $f$ and $\delta$ satisfy the conditions of Lemma 2.3, so that, by the maximality of 
$A,(A, f)=A$. Hence $g^{1 / 2}=2\left\|g^{1 / 2}\right\| f \in A$. Similarly, it can be shown in turn that $g^{1 / 4}, g^{1 / 8}, \cdots$ all belong to $A$. Since any positive real $\lambda$ can be approximated by the sum of an integer and various powers of $\frac{1}{2}$, and since $A$ is closed, it follows that $g^{\lambda} \in A(\forall \lambda>0)$. If, now, $h$ is any element in $A$, then for each positive integer $n,\left(h+n^{-1}\right) \in A \cap C^{*}(E)$, so that $\left(h+n^{-1}\right)^{\lambda} \in A \quad(\forall \lambda>0)$. Taking the limit as $n \rightarrow \infty$, we see that $h^{\lambda} \in A$. Thus $A$ is a cornet, and, being maximal, must, by Theorem 2.1 Corollary 2 , be geometric.

\section{REFERENCES}

1. F. F. Bonsall, Semi-algebras of continuous functions, Proc. Internat. Sympos. Linear Spaces, Jerusalem, 1960, 101-114.

2. - Algebraic properties of some convex cones of functions, Quart. J. Math. Oxford Ser. (2) 14 (1963), 225-230.

3. - Locally compact semi-algebras Proc., London Math. Soc. (3) 13 (1963), 51-70.

4. N. Bourbaki, Intégration, Chapters I-IV, Actualités Sci. Ind. No. 1175, Hermann, Paris, 1952.

5. D. G. Bourgin, Multiplicative transformations, Proc. Nat. Acad. Sci. U.S.A. 36 (1950), 564-570.

6. S. Bourne, On normed semi-algebras, Studia Math. 21 (1961), 45-54.

7. G. Choquet and J. Deny, Ensembles semi-réticulés et ensembles réticulés de fonctions continues, J. Math. Pures Appl. 36 (1957), 179-189.

8. M. M. Day, Normed linear spaces, (reprint), Springer, Berlin, 1962.

9. G. H. Hardy, J. E. Littlewood and G. Pólya, Inequalities, Cambridge Univ. Press, New York, 1934.

10. K. Hoffman and I. M. Singer, Maximal algebras of continuous functions, Acta Math. 103 (1960), 217-241.

11. W. B. Jurkat and G. G. Lorentz, Uniform approximation by polynomials with positive coefficients, Duke Math. J. 28 (1961), 463-473.

12. R. V. Kadison, A representation theory for commutative topological algebra, Mem. Amer. Math. Soc. No. 7 (1951), 39 pp.

13. J. F. C. Kingman, A convexity property of positive matrices, Quart. J. Math. Oxford Ser. (2) 12 (1961), 283-284.

14. F. Riesz, Sur quelques notions fondamentales dans la théorie générale des opérations linéaires, Ann. of Math. 41 (1940), 175-206.

THE UNIVERSITY,

NEWCASTLE-UPON-TYNe, ENGLAND

THE UNIVERSITY OF WESTERN ONTARIO,

LONDON, CANADA 